International Journal of Modern Physics B, (C) World Scientific Publishing Company

\title{
FORWARD ELECTRON-PHONON SCATTERING IN NORMAL AND SUPERCONDUCTING STATES
}

\author{
O.V.DOLGOV \\ Institut für Theoretische Physik, Universität Tübingen \\ Tübingen, Germany \\ O.V.DANYLENKO \\ P. N. Lebedev Physical Institute, Moscow, Russia \\ M. L. KULIĆ \\ Centre de Physique Théorique et de Modélisation, Université Bordeaux I, France \\ V. OUDOVENKO \\ Joint Institute for Nuclear Research, Dubna, Russia
}

\begin{abstract}
The sharp forward electron-phonon $(F E P)$ and impurity $(F I S)$ scattering change the normal and superconducting properties significantly. The pseudo-gap like features are present in the density of states for $\omega<\Omega$, where $\Omega$ is the phonon frequency. The superconducting critical temperature $T_{c}$, due to the $F E P$ pairing, is linear with respect to the electron-phonon coupling constant. The FIS impurities are pair weakening for $s-$ and $d-$ wave pairing.
\end{abstract}

\section{Introduction}

There is growing experimental evidence for $\mathrm{d}$-wave pairing in high- $\mathrm{T}_{c}$ superconductors (HTS), which are seemingly in contradiction with the standard phonon mechanism of pairing. However, some optic and tunneling measurements show features which can be ascribed to rather strong electron-phonon (E-P) coupling. The idea of d-wave pairing in HTS oxides due to the renormalized (by strong electronic correlations) E-P coupling has been put forward in Ref.[1]. It was shownt that for small hole doping, strong Coulomb correlations renormalize the E-P interaction giving rise to the strong forward (small-q) scattering peak. This renormalization means that each quasiparticle, due to the suppression of the doubly occupancy on the same lattice, is surrounded by a giant correlation hole. The pronounced forward E-P scattering can be also due to the poor Coulomb screening in HTS oxides as well as due to the large density of states near some points at the Fermi surface 2 . In the following analysis we assume an extreme case of the forward electron-phonon interaction (FEP pairing) and of the forward nonmagnetic impurity scattering (FIS impurities), i.e. that $\left|g_{s c r}(\vec{q})\right|^{2} \sim \delta(\vec{q})$ and $u_{s c r}^{2}(\vec{q}) \sim \delta(\vec{q})$, where $\delta(\vec{q})$ is the Dirac delta-function, $g_{s c r}(\vec{q})$ and $u_{s c r}(\vec{q})$ are the screened E-P and impurity potentials respectively. This approximation picks up the main physics, and it is valid whenever the range $R$ of $\left|g_{s c r}(\vec{q})\right|^{2}$ and $u_{s c r}^{2}(\vec{q})$ fulfils the condition $R \gg k_{F}^{-1}$, i.e. $q_{c} \ll k_{F}$. 
Moreover, it greatly simplifies the structure of the Eliashberg equations by omitting integration in $\vec{k}$-space. A similar approximation was successfuly used for the $\mathrm{AF}$ spin-fluctuation mechanism of pairing, where the four peaks at $\vec{Q}=( \pm \pi, \pm \pi)$ in the spin-fluctuation density were replaced by delta-functions 3 .

\section{Eliashberg equations for FEP pairing and FIS impurities}

The Eliashberg equations in the presence of the FEP pairing potential $\delta(\vec{k}) V_{e p}(\omega)$ and of the FIS impurities $\delta(\vec{k}) u_{i m p}^{2}$, where the latter is treated first in the selfconsistent Born approximation. The renormalization function $Z$, the energy renormalization $\bar{\xi}$ and the superconducting order parameter $\Delta$ are solutions of the following equations

$$
\begin{gathered}
Z_{n}(\xi)=1+\frac{T}{\omega_{n}} \sum_{m} V_{e f f}(n-m) \frac{\omega_{m} Z_{m}(\xi)}{\left[\omega_{m} Z_{m}(\xi)\right]^{2}+\bar{\xi}_{m}^{2}(\xi)+\left[Z_{m}(\xi) \Delta_{m}(\xi)\right]^{2}} \\
\bar{\xi}_{n}(\xi)=\xi(\vec{k})-T \sum_{m} \frac{V_{e f f}(n-m) \bar{\xi}_{m}(\xi)}{\left[\omega_{m} Z_{m}(\xi)\right]^{2}+\bar{\xi}_{m}^{2}(\xi)+\left[Z_{m}(\xi) \Delta_{m}(\xi)\right]^{2}} \\
Z_{n}(\xi) \Delta_{n}(\xi)=T \sum_{m} \frac{V_{e f f}(n-m) Z_{m}(\xi) \Delta_{m}(\xi)}{\left[\omega_{m} Z_{m}(\xi)\right]^{2}+\bar{\xi}_{m}^{2}(\xi)+\left[Z_{n}(\xi) \Delta_{m}(\xi)\right]^{2}}
\end{gathered}
$$

The retardation (strong-coupling) effects are considered in the Einstein model with one phonon at a frequency $\Omega$. The solution of Eqs.(1) can be found by iteration, where the first iteration step at $T=0$ and the analytical continuation give $\Re Z(\omega, \xi)=1+\frac{\lambda}{2 N(0)} \frac{\Omega}{(\Omega+|\xi|)^{2}-\omega^{2}}$, and $\Im Z(\omega, \xi)=-\frac{\pi \lambda}{4 N(0)} \delta(\omega \pm(\Omega+|\xi|))$. In contrast to the standard behaviour, where the spectral function $A(\omega, \xi)$ has a coherent peak at $\omega= \pm|\xi|$ and incoherent wings for $\omega>\Omega$, the $F E P$ interaction leads to a rather broad peak at $\omega= \pm|\xi|$ and very sharp peaks at $|\xi| \pm \Omega$. As a consequence the density of states $N(\omega)$ obtained by the solution of Eqs.(1) (see Fig.1) shows a maximum at $\omega=0$, which narrows by lowering $T$, and pseudogap like behavior below $\Omega$. The pseudogap feature disappears at $T$ comparable with the phonon energy $\Omega$.

\section{Superconductivity due to FEP}

In the weak coupling limit one obtains $Z(\vec{k}, n)=1$ and the $F E P$ pairing gives the maximum $T_{c}$ on the Fermi surface, i.e. for $\xi=0$ where $\bar{\xi}_{n}(\vec{k}, n)=0$. The solution of Eqs.(1) in the weak coupling limit and for $T_{c} \ll \Omega$ is given by $T_{c 0}=$ $\lambda / 4 N(0)$, where $\lambda=N(0) V_{e p}$. Several points should be stressed: first, $T_{c 0}$ is linear in $\lambda$ in the case of FEP pairing, similar to Ref.3. Second, in the case of the electron-phonon pairing potential $V_{e p}\left(\vec{q}, \omega_{n}\right)$ with a finite cut-off $\left(q_{c} \neq 0\right)$ it is shown in Ref.4 that $T_{c 0} \equiv T_{c 0}\left(q_{c}=0\right)$ is the value of $T_{c}$ zeroth-order in $q_{c}$. For the short-range pairing potential $\left(q_{c} \sim 2 k_{F}\right)$, i.e. when $q_{c} V_{F} \sim W \sim 1 / N(0)$, one obtains the standard BCS result $T_{c 0}^{B C S}=1.13 \Omega \exp (-1 / \lambda)$, while for the longrange pairing potential $\left(q_{c} V_{F} \ll \Omega\right)$ the finite- $q_{c}$ correction to $T_{c 0}$ is given by $T_{c} \simeq$ $T_{c 0}\left(1-7 \zeta(3) q_{c} V_{F} / 4 \pi^{2} T_{c 0}\right)$. 


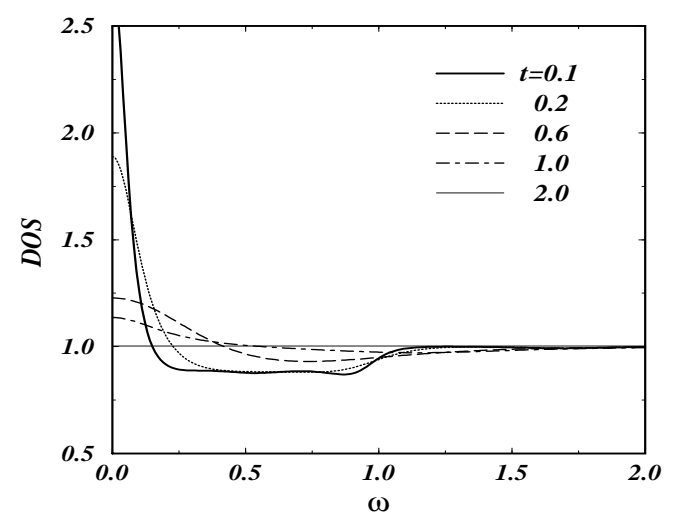

Fig. 1. - The density of states $N(\omega)$ for the $F E P$ scattering with the given values of $t \equiv \pi T / \Omega$.

The FS impurities affect $T_{c}$, which is due to the FEP pairing. Some limiting cases are considered: (a) $\underline{\Gamma_{F} \ll \pi T_{c}}$ : One obtains $T_{c}=T_{c 0}\left[1-4 \Gamma_{F} / 49 T_{c 0}\right]$; (b) $\underline{\Gamma_{F} \gg \pi T_{c}}$ : If $\Gamma_{F} \gg \Omega / 2$ is fulfilled one obtains $T_{c} \approx(\pi / 2 \gamma) \Omega \exp \left(-\pi \Gamma_{F} / V_{e p}\right)$. Note that the $F I S$ impurities are pair weakening for the FEP pairing, i.e. there is an exponential fall-off of $T_{c}$ with the increase of $\Gamma_{F}$. One can show that the NIS impurities are pair weakening for $s$-wave- and pair breaking for $d$-wave FEP pairing

\section{Conclusions}

In summary, it is shown here that: $(a)$ the FEP interaction and the FIS scattering change the quasiparticle spectral properties significantly. In the case of the $F E P$ interaction there are pseudogap features in the density of states $N(\omega)$ for $\omega<\Omega$ (phonon frequency); (b) by assuming that the pairing is due to the forward E-P $(F E P)$ scattering the critical temperature of clean systems $T_{c 0}$ depends linearly on the E-P coupling constant $\lambda$ in the Migdal approximation; $(c)$ the FIS impurities affect in the same way the $s$ - and $d$-wave FEP pairing and they are pair-weakening for both pairings; $(d)$ the NIS isotropic impurities are pair-weakening for the $s$ wave FEP pairing and pair-breaking for the $d$-wave FEP pairing.

1. M. Kulić and R. Zeyher, Phys. Rev. B 49, 4395 (1994); R. Zeyher and M. Kulić, ibid 53, 2850 (1996); ibid 54, 8985 (1996)

2. A. A. Abrikosov, Physica C 244, 243 (1995); J. Ruvalds et al., Phys. Rev. B 51, 3797 (1995); M. Weger et al., J. Low Temp. Phys. 95, 131 (1994); G. Santi et al., J. of Supercond. 8, 215 (1995); L. Pietronero, S. Strässler and C. Grimaldi, Phys. Rev. B 52, 10516 (1995); ibid 52, 10530 (1995)

3. V. N. Kostur, B. Mitrović, Phys. Rev. B 50, 12774 (1994); ibid 51, 6064 (1995)

4. O. V. Danylenko, O. V. Dolgov, M. L. Kulić, V. Oudovenko, cond-mat/9710234. 